\title{
CUBIC DIOPHANTINE INEQUALITIES
}

\author{
R. C. BAKER, J. BRÜDERN AND T. D. WOOLEY
}

§1. Introduction. Let $\lambda_{1}, \ldots, \lambda_{s}$ be nonzero real numbers and suppose that $\lambda_{1} / \lambda_{2}$ is irrational. In 1955 , Davenport and Roth showed [6] that the values taken by

$$
\lambda_{1} x_{1}^{3}+\ldots+\lambda_{s} x_{s}^{3}
$$

at integer points $\left(x_{1}, \ldots, x_{s}\right)$ are dense on the real line, provided that $s \geqslant 8$. In the present paper we obtain the same result with seven variables.

THEOREM. Let $\sigma=10^{-4}$. Let $\lambda_{1}, \ldots, \lambda_{7}$ be non-zero real numbers with $\lambda_{1} / \lambda_{2}$ irrational. For each real $\mu$, the inequality

$$
\left|\lambda_{1} x_{1}^{3}+\ldots+\lambda_{7} x_{7}^{3}+\mu\right|<\left(\max _{j}\left|x_{j}\right|\right)^{-\sigma}
$$

has infinitely many solutions in integers.

The result is somewhat analogous with Linnik's theorem [7] that $R_{7,3}(n)$, the number of representations of $n$ as the sum of seven cubes, is positive for large $n$. However, a proof of the above theorem only became a feasible prospect with the appearance of Vaughan's work $[10,11,12]$. Vaughan used the HardyLittlewood method to give a good lower bound for $R_{7,3}(n)$ [10], and then a lower bound of the expected order of magnitude [12], that is,

$$
R_{7,3}(n) \gg n^{4 / 3}
$$

for large $n$. The method of Davenport and Roth is a variant of the HardyLittlewood method. However, the bounds for even moments of smooth Weyl sums in Vaughan [11] 'just miss' what one would need to initiate a proof of the Theorem. Recently Wooley [13] gave sharper bounds for $s$-th power moments of such sums, for all real $s>4$. These new moment estimates are the key element in our proof.

We introduce some notation concerning smooth Weyl sums. Denote by $\mathscr{A}(P, R)$ the set of $R$-smooth numbers of size at most $P$, that is,

$$
\mathscr{A}(P, R)=\{n \in[1, P] \cap \mathbb{Z}: p \mid n \Rightarrow p \leqslant R\} .
$$

Here and subsequently, $p, p_{1}, p_{2}, \ldots$ denote prime numbers. Let $k$ be a fixed integer, $k \geqslant 3$. We define the smooth Weyl sum $f(\alpha)=f(\alpha ; P, R)$ by

$$
f(\alpha ; P, R)=\sum_{x \in, \alpha(P, R)} e\left(\alpha x^{k}\right)
$$


where $e(z)$ denotes $e^{2 \pi i z}$. Let

$$
U_{s}(P, R)=\int_{0}^{1}|f(\alpha ; P, R)|^{s} d \alpha .
$$

We shall say that an exponent $\mu_{s}=\mu_{s, k}$ is permissible whenever the exponent has the property that, for each $\varepsilon>0$, there exists a positive number $\eta=$ $\eta(\varepsilon, s, k)$ such that whenever $R \leqslant P^{\eta}$, one has

$$
U_{s}(P, R) \ll_{\varepsilon, s, k} P^{\mu_{s, k}+\varepsilon} .
$$

In order to prove our Theorem we need the following results about moments.

Proposition 1. Let $\xi$ be the positive root of the polynomial

$$
\xi^{3}+16 \xi^{2}+28 \xi-8
$$

so that $\xi=0.2495681 \ldots$ Then $\mu_{6,3}=3+\xi$ is a permissible exponent.

PROPOSITION 2. The exponent $\mu_{20 / 3,3}=3.7941603$ is permissible.

For comparison, Vaughan [11, Theorem 4.4] showed that $\mu_{6,3}=3.25$ is permissible; one would obtain the permissible expohent $\mu_{20 / 3,3}=3.83$ on combining this with Hölder's inequality and Hua's inequality for the eighth moment.

Proposition 1 is a corollary of [13, Theorem 1.2]. We shall prove Proposition 2 in Section 2. Owing to the highly iterative nature of the method of [13], we are forced to calculate a number of intermediate moments. In compensation, the estimates provided below for each of these moments appear to be the best deriving from currently available arguments.

Once we have these Propositions, and some further lemmata on Weyl sums which we assemble in Section 3, we are able to proceed with the proof of the Theorem in a relatively straightforward fashion. Section 4 reduces the problem to the estimation of an integral over the real line and deals with the major arc. This is familiar ground; compare Brüdern [3]. In Section 5, we discuss the minor arc. Proposition 1 yields some initial Diophantine approximation, and Proposition 2 can then be used to dispose of the most difficult case.

\$2. The 20/3-moment. It is convenient to describe an exponent $\delta_{s}=\delta_{s, k}$ as associated if the exponent

$$
\mu_{s}=s / 2+\delta_{s}
$$

is permissible in the notation of Section 1 .

When $k=3$ and $s<5$ we are able to calculate the permissible exponents $\mu_{s}$ by using a simple corollary of $[13$, Theorem 1.1] which we summarize below in the form of a lemma. 
LEMMA 1. Let $s$ be a real number with $s>2$, and suppose that $\delta_{s, 3}$ and $\delta_{2 s, 3}$ are associated exponents. Then the exponent $\delta_{s+2,3}$ is associated, where

$$
\delta_{s+2}=\delta_{s}(1-\theta)+\frac{1}{2} s \theta
$$

and

$$
\theta=\frac{\delta_{2 s}-2 \delta_{s}}{4+\delta_{2 s}-2 \delta_{s}}
$$

Proof. We set $k=3$ and $t=1$ in [13, Theorem 1.1] and note equation (2.1) above.

When $k=3$ and $s>6$ one obtains superior permissible exponents by employing an estimate of [13, Lemma 3.4] within the argument of $[13, \S 4]$.

LemMa 2. Define the integer $\tau=\tau(k) b y$

$$
\tau(k)= \begin{cases}1, & \text { when } k=3, \text { and when } k \geqslant 8 \text { and } k \text { is even } \\ 0, & \text { otherwise. }\end{cases}
$$

Let $s$ and $t$ be real numbers with $0<t \leqslant 1$ and $s+2 t>4$. Write $v=s(1-t / 4)^{-1}$. Suppose that $\mu_{s, k}$ and $\mu_{v, k}$ are permissible exponents. Then $\mu_{s+2 t, k}$ is permissible, where

$$
\mu_{s+2 t}=\mu_{s}(1-\theta)+t+s \theta
$$

and

$$
\theta=\frac{t+(4-t) \mu_{v}-4 \mu_{s}}{3 t k+t(1-\tau)+(4-t) \mu_{v}-4 \mu_{s}}
$$

Proof. Suppose that $u$ and $t$ are real numbers with $0<t \leqslant 1$ and $u+2 t>4$, and write $s=u+2 t$. Take $\varphi$ to be a real number with $0 \leqslant \varphi \leqslant 1 / k$ to be chosen later, and write

$$
M=P^{\varphi}, \quad H=P M^{-k} \quad \text { and } \quad Q=P M^{-1} .
$$

We apply the argument of $[13, \S 4]$, setting $v=u(1-t / 4)^{-1}$ in the application of Lemma 3.4 of that paper. We may suppose that $\mu_{u, k}$ and $\mu_{v, k}$ are permissible exponents. Then as in [13, \$4] (see in particular (4.2) and (4.3)), our choice for $\varphi$ is

$$
\varphi=\min (\theta, 1 / k)
$$

where

$$
\theta=\frac{t+(4-t) \mu_{v}-4 \mu_{u}}{3 t k+t(1-\tau)+(4-t) \mu_{v}-4 \mu_{u}}
$$


We may now mimic the argument of the proof of Theorem 1.1 in $\$ 4$ of [13] to deduce that

$$
\mu_{s}^{*}=\mu_{u}(1-\theta)+t+u \theta
$$

is permissible, from which the lemma follows immediately.

In the remainder of the paper, we assume that $k=3$.

COROLlary. Let $s$ be a real number with $s>2$, and suppose that $\delta_{s, 3}$ and $\delta_{4 s / 3,3}$ are associated exponents. Then the exponent $\delta_{s+2,3}$ is associated, where

$$
\delta_{s+2}=\delta_{s}(1-\theta)+\frac{1}{2} s \theta
$$

and

$$
\theta=\frac{1+3 \delta_{4 s / 3}-4 \delta_{s}}{9+3 \delta_{4 s / 3}-4 \delta_{s}}
$$

Proof. We set $k=3$ and $t=1$ in Lemma 2, and note equation (2.1).

Proof of Proposition 2. We estimate the 20/3-moment in steps (a)-(h) below.

(a) $0<s \leqslant 4$. By combining [13, Lemma 2.1] with $(2.1)$, we find that the exponent $\delta_{s}=0$ is associated.

(b) $s=5,6$. The proof of [13, Theorem 1.2] shows that the exponents $\delta_{6}=$ 0.2495682 and $\delta_{5}=0.0880919$ are associated.

(c) $s=9 / 2$. We apply Lemma 1 with $s=5 / 2$. Thus we deduce from (a) that whenever $\delta_{5}$ is an associated exponent, then so is

$$
\delta_{9 / 2}=\frac{5 \delta_{5}}{4\left(4+\delta_{5}\right)} .
$$

Consequently, from (b), we deduce that $\delta_{9 / 2}=0.0269356$ is an associated exponent.

(d) $s=16 / 3$. We apply Hölder's inequality to interpolate linearly between available associated exponents $\delta_{5}$ and $\delta_{6}$. Thus, whenever $\delta_{5}$ and $\delta_{6}$ are associated exponents, then so is

$$
\delta_{16 / 3}=\frac{2}{3} \delta_{5}+\frac{1}{3} \delta_{6} .
$$

It therefore follows from (b) that $\delta_{16 / 3}=0 \cdot 1419174$ is an associated exponent.

(e) $s=14 / 3$. We apply Lemma 1 with $s=8 / 3$. Thus we deduce from (a) that whenever $\delta_{16 / 3}$ is an associated exponent, then so is

$$
\delta_{14 / 3}=\frac{4 \delta_{16 / 3}}{3\left(4+\delta_{16 / 3}\right)} \text {. }
$$

Thus, in view of (d), we find that $\delta_{14 / 3}=0.0456850$ is an associated exponent. 
(f) $s=13 / 2$. We apply the Corollary with $s=9 / 2$. Then we deduce that whenever $\delta_{9 / 2}$ and $\delta_{6}$ are associated exponents, then so is

$$
\delta_{13 / 2}=\delta_{9 / 2}(1-\theta)+\frac{9 \theta}{4}
$$

where

$$
\theta=\frac{1+3 \delta_{6}-4 \delta_{9 / 2}}{9+3 \delta_{6}-4 \delta_{9 / 2}}
$$

Consequently, it follows from (b) and (c) that $\delta_{13 / 2}=0.4053175$ is an associated exponent.

(g) $s=56 / 9$. We aply Hölder's inequality to interpolate linearly between available associated exponents $\delta_{6}$ and $\delta_{13 / 2}$. Thus, whenever $\delta_{6}$ and $\delta_{13 / 2}$ are associated exponents, then so is

$$
\delta_{56 / 9}=\frac{5}{9} \delta_{6}+\frac{4}{9} \delta_{13 / 2} \text {. }
$$

Thus we find from (b) and (f) that $\delta_{56 / 9}=0.3187902$ is an associated exponent.

(h) $s=20 / 3$. We apply the Corollary with $s=14 / 3$. Thus we deduce that whenever $\delta_{14 / 3}$ and $\delta_{56 / 9}$ are associated exponents, then so is

$$
\delta_{20 / 3}=\delta_{14 / 3}(1-\theta)+\frac{7}{3} \theta
$$

where

$$
\theta=\frac{1+3 \delta_{56 / 9}-4 \delta_{14 / 3}}{9+3 \delta_{56 / 9}-4 \delta_{14 / 3}}
$$

Consequently, from (e) and (g), it follows that $\delta_{20 / 3}=0.4608269$ is an associated exponent.

Proposition 2 now follows from (h) on recalling (2.1).

§3. Lemmata on Weyl sums. Let $\varepsilon$ be a sufficiently small positive constant and let $\eta$ be sufficiently small as a function of $\varepsilon$. Constants implicit in '«' or ' $O$ ' notation will depend at most on $\varepsilon$. If

$$
A \ll B \ll A,
$$

we write $\alpha \asymp B$.

LEMMA 3. Let $N=465767$,

$$
\begin{array}{ll}
\theta_{1}=38416 N^{-1}, & \theta_{2}=41160 N^{-1}, \\
\theta_{4}=51506 N^{-1}, & \theta_{5}=58521 N^{-1},
\end{array}
$$


and define $g(\alpha)=g(P ; \alpha)$ by

$$
g(P ; \alpha)=\sum_{\substack{P^{\theta_{i}} \\\left(1 \leqslant p_{i} \leqslant 2 P^{\theta_{i}}\right.}} \sum_{P /\left(p_{1} \ldots p_{5}\right)<y \leqslant 2 P /\left(p_{1} \ldots p_{5}\right)} e\left(\alpha\left(p_{1} \ldots p_{5} y\right)^{3}\right) .
$$

Then

$$
\int_{0}^{1}|g(P ; \alpha)|^{6} d \alpha \ll P^{3+2 \theta_{5}+\varepsilon}
$$

Proof. This is (a trivial variant of) the case $\ell=5$ of Brüdern [4].

Notice that the exponent in Lemma 3 is a little worse than Vaughan's value $\mu_{6,3}=3.25$ mentioned in Section 1. In compensation we have Lemma 5 below, where we make good use of the long inner summation over $y$. Lemma 4 is the stronger counterpart for an 'ordinary' Weyl sum.

LemMA 4. Let $A \geqslant P^{3 / 4 \star . \varepsilon}$. Suppose that

$$
\left|\sum_{P<x \leqslant 2 P} e\left(\beta x^{3}\right)\right| \geqslant A \text {. }
$$

Then there are coprime integers $b, r$ such that

$$
\begin{gathered}
1 \leqslant r \ll P^{3} A^{-3} \\
|\beta-b / r| \ll r^{-1 / 3} A^{-1} P^{-2} .
\end{gathered}
$$

Proof. See for example the Lemma in [1], \$4.

Lemma 5. Let $B \gg P^{8 / 9}$. Let $g$ be as in Lemma 3. If $|g(P ; \beta)| \geqslant B$ then there are coprime $a, q$ with

$$
\begin{gathered}
1 \leqslant q \ll P^{3} B^{-3} \\
|\beta-a / q| \ll q^{-1 / 3} B^{-1} P^{-2} .
\end{gathered}
$$

Proof. Let

$$
\begin{gathered}
\mathscr{M}=\left\{p_{1} \ldots p_{5}: P^{\theta_{i}}<p_{i} \leqslant 2 P^{\theta_{i}}\right\}, \\
k(\alpha, Z)=\sum_{Z<z \leqslant 2 Z} e\left(\alpha z^{3}\right) .
\end{gathered}
$$

We have

$$
g(\alpha)=\sum_{m \in \mathscr{M}} k\left(\alpha m^{3}, P / m\right)
$$

We write $\theta_{0}=1-\theta_{1}-\theta_{2}-\ldots-\theta_{5}=0.494 \ldots$ and $Y=P^{\theta_{0}}$. For $m \in \mathscr{M}$ we have $P / m \asymp Y$. 
Given $m \in \mathscr{M}$, we may choose coprime $b, r$ with $1 \leqslant r \leqslant 24 Y^{2}$,

$$
\left|a m^{3}-b / r\right| \leqslant(24 r)^{-1} Y^{-2} \text {. }
$$

If $r>Y$ then, by Weyl's inequality,

$$
k\left(\alpha m^{3}, P / m\right) \ll Y^{3 / 4+\varepsilon}
$$

while for $r \leqslant Y$,

$$
k\left(\alpha m^{3}, P / m\right) \ll r^{-1 / 3} Y\left(1+Y^{3}\left|\alpha m^{3}-\frac{b}{r}\right|\right)^{-1}+r^{1 / 2+\varepsilon} .
$$

This follows at once from [8, Lemma 6.1]. We see that

$$
k\left(\alpha m^{3}, P / m\right) \ll Y^{3 / 4+\varepsilon}
$$

unless

$$
1 \leqslant r \leqslant Y^{3 / 4} ; \quad\left|\alpha m^{3}-b / r\right| \leqslant \frac{1}{2} r^{-1} Y^{-9 / 4}
$$

Consequently,

$$
B \leqslant|g(\alpha)| \ll Y^{3 / 4+\varepsilon} P^{\theta_{1}+\ldots+\theta_{5}}+\sum_{m \in \mathscr{M}^{\prime}} r^{-1 / 3} Y\left(1+Y^{3}\left|\alpha m^{3}-\frac{b}{r}\right|\right)^{-1}
$$

where $\mathscr{M}^{\prime}$ is the subset of $\mathscr{M}$ such that (3.1) holds. But

$$
Y^{3 / 4+\varepsilon} P^{\theta_{1}+\ldots+\theta_{5}} \ll P^{1+\varepsilon} Y^{-1 / 4} \ll P^{8 / 9-\varepsilon},
$$

so that

$$
B \ll \sum_{m \in N^{\prime}} r^{-1 / 3} Y\left(1+Y^{3}\left|\alpha m^{3}-\frac{b}{r}\right|\right)^{-1} .
$$

For convenience write $m=m_{1} m_{2}$ with $m_{1}=p_{1} p_{2} p_{3}, m_{2}=p_{4} p_{5}$. For any $m=$ $m_{1} m_{2} \in \mathscr{M}^{\prime}$ we may choose coprime $c, s$ (depending only on $m_{1}$ ) such that

$$
\left|\alpha m_{1}^{3}-\frac{c}{s}\right| \leqslant \frac{1}{s Y^{9 / 4}}, \quad s \leqslant Y^{9 / 4}
$$

Then

$$
\left|\alpha m^{3}-\frac{c m_{2}^{3}}{s}\right| \leqslant \frac{m_{2}^{3}}{s Y^{9 / 4}}
$$

so that

$$
\begin{aligned}
\left|\frac{c m_{2}^{3}}{s}-\frac{b}{r}\right| & \leqslant \frac{m_{2}^{3}}{s Y^{9 / 4}}+\frac{1}{2} r^{-1} Y^{-9 / 4}, \\
\left|c m_{2}^{3} r-b s\right| & \leqslant m_{2}^{3} r Y^{-9 / 4}+\frac{1}{2} s Y^{-9 / 4} \\
& \leqslant 64 P^{3\left(\theta_{4}+\theta_{s}\right)-3 \theta_{0} / 2}+\frac{1}{2}<1 .
\end{aligned}
$$


It follows that $r=s\left(s, m_{2}^{3}\right)^{-1}$, and hence

$$
\begin{aligned}
B & \ll Y \sum_{m_{1} m_{2} \in \mathscr{M}^{\prime}} s^{-1 / 3}\left(s, m_{2}^{3}\right)^{1 / 3}\left(1+Y^{3} m_{2}^{3}\left|\alpha m_{1}^{3}-c / s\right|\right)^{-1} \\
& \ll Y P^{\theta_{4}+\theta_{5}} \sum_{m_{1}} s^{-1 / 3}\left(1+Y^{3} P^{3\left(\theta_{4}+\theta_{5}\right)}\left|\alpha m_{1}^{3}-c / s\right|\right)^{-1} .
\end{aligned}
$$

The last sum extends over $m_{1}=p_{1} p_{2} p_{3}\left(P^{\theta_{i}}<p_{i} \leqslant 2 P^{\theta_{i}}\right)$.

We now repeat this line of argument. The sum in (3.2) may be restricted to those $m_{1}$ for which

$$
\begin{gathered}
1 \leqslant s \ll P^{1 / 3}, \\
\left|\alpha m_{1}^{3}-c / s\right|<P^{1 / 9-3\left(\theta_{4}+\theta_{5}\right)+\varepsilon} Y^{-3} .
\end{gathered}
$$

We now choose coprime $a, q$,

$$
1 \leqslant q \leqslant P^{3 / 2}, \quad\left|\alpha-\frac{a}{q}\right| \leqslant \frac{1}{q P^{3 / 2}} .
$$

For those $m_{1}$ satisfying (3.3), (3.4),

$$
\begin{gathered}
\left|\frac{c}{s}-\frac{a m_{1}^{3}}{q}\right|<P^{1 / 9-3\left(\theta_{4}+\theta_{5}\right)+\varepsilon} Y^{-3}+\frac{m_{1}^{3}}{q P^{3 / 2}}, \\
\left|c q-a m_{1}^{3} s\right|<s P^{3 / 2+1 / 9-3\left(\theta_{4}+\theta_{5}\right)+\varepsilon} Y^{-3}+s m_{1}^{3} P^{-3 / 2}<1
\end{gathered}
$$

after a short computation. Thus $s=q /\left(q, m_{1}^{3}\right)$ and

$$
\begin{aligned}
B & \ll Y P^{\theta_{4}+\theta_{5}} \sum_{m_{1}} q^{-1 / 3}\left(q, m_{1}^{3}\right)^{1 / 3}\left(1+P^{3}\left|\alpha-\frac{a}{q}\right|\right)^{-1} \\
& \ll P q^{-1 / 3}\left(1+P^{3}\left|\alpha-\frac{\alpha}{q}\right|\right)^{-1} .
\end{aligned}
$$

The lemma follows at once.

LEMMA 6. Let

$$
f(\alpha)=\sum_{P<x \leqslant 2 P} e\left(\alpha x^{3}\right)
$$

Let

$$
\mathscr{F}=\left\{\alpha \in[0,1]:|f(\alpha)|>P^{3 / 4+\varepsilon}\right\} .
$$

Then

$$
\int_{\mathscr{F}}|f(\alpha)|^{4} d \alpha \ll P^{1+\varepsilon}
$$

Proof. See for example Brüdern [2], proof of (4.6). 
LEMMA 7. Let $1 \leqslant B \leqslant P^{1 / 4-\varepsilon}$. Let $\mathscr{E}(B)=\left\{\alpha \in[0,1]:|f(\alpha)| \geqslant P B^{-1}\right\}$. Then

$$
\int_{\mathscr{E}(B)}|f(\alpha)|^{3} d \alpha \ll P^{\varepsilon} B
$$

Proof. By Lemma 6,

$$
\int_{\mathscr{B}(B)}|f|^{3} d \alpha \leqslant P^{-1} B \int_{\mathscr{F}}|f|^{4} d \alpha \ll P^{\varepsilon} B
$$

\$4. The Davenport-Heilbronn method. According to Davenport [5] for every integer $r$ there is a function $K: \mathbb{R} \rightarrow \mathbb{R}$ with

$$
K(-\alpha)=K(\alpha), \quad K(\alpha)<C(r) \min \left(1,|\alpha|^{-r}\right)
$$

whose Fourier transform satisfies

$$
\hat{K}(\alpha)= \begin{cases}1, & |\alpha| \leqslant 1 / 3 \\ 0, & |\alpha| \geqslant 1\end{cases}
$$

and $0 \leqslant \hat{K}(\alpha) \leqslant 1$ for $1 / 3<|\alpha| \leqslant 1$.

In proving our Theorem we may assume $\lambda_{1}>0, \lambda_{2}<0$ (change $x_{j}$ into $-x_{j}$ if necessary). Let

$$
\begin{aligned}
& f_{1}(\alpha)=\sum_{P \lambda_{1}^{-1 / 3}<x \leqslant 2 P \lambda_{1}^{-1 / 3}} e\left(\alpha \lambda_{1} x^{3}\right), \\
& g_{2}(\alpha)=g\left(P\left|\lambda_{2}\right|^{-1 / 3}, \lambda_{2}\right)
\end{aligned}
$$

(in the notation of Lemma 3), and

$$
h_{j}(\alpha)=\sum_{y \in, \alpha\left(X, X^{\eta}\right)} e\left(\alpha \lambda_{j} y^{3}\right)
$$

where $X=P / \log P$. Now consider

$$
\mathscr{N}(P)=\int_{-\infty}^{\infty} f_{1}(\alpha) g_{2}(\alpha) h_{3}(\alpha) \ldots h_{7}(\alpha) e(\alpha \mu) K\left(\alpha P^{-\sigma}\right) d \alpha
$$

By a familiar argument (see e.g. [3], §3), it suffices to prove that $\mathcal{N}(P) \rightarrow \infty$ as $P$ runs through some sequence of positive numbers tending to infinity.

Let

$$
\begin{aligned}
& \mathscr{M}=\left\{\alpha:|\alpha| \leqslant P^{-2-\varepsilon}\right\}, \\
& m=\left\{\alpha: P^{-2-\varepsilon}<|\alpha| \leqslant P^{\sigma+\varepsilon}\right\}, \\
& \mathscr{T}=\left\{\alpha:|\alpha|>P^{\sigma+\varepsilon}\right\} .
\end{aligned}
$$

It is easy to see that for a suitable choice of $r=r(\varepsilon)$, the contribution of $\mathscr{T}$ to $\mathcal{N}(P)$ is $O(1)$; compare [3], p. 54. 
On the 'major arc' $\mathscr{M}$ we can give satisfactory approximations to $f_{1}$ and $g_{2}$. Let

$$
v(\beta, P)=\int_{B}^{2 B} e\left(\beta \gamma^{3}\right) d \gamma .
$$

Then, for $\alpha \in \mathscr{M}$,

$$
\begin{aligned}
f_{1}(\alpha) & =v\left(\lambda_{1} \alpha, P \lambda_{1}^{-1 / 3}\right)+O(1) \\
& =\lambda_{1}^{-1 / 3} v(\alpha, P)+O(1),
\end{aligned}
$$

by Theorem 4.1 of Vaughan [8]. Similarly, for

$$
|\alpha| \leqslant P^{-5 / 2} \text {, }
$$

Theorem 2 of Vaughan [9] yields

$$
\begin{aligned}
g_{2}(\alpha) & =\sum_{\substack{\theta_{i}<p_{i} \leqslant 2 p^{\theta_{i}} \\
(1 \leqslant i \leqslant 5)}}\left(v\left(\lambda_{2} p_{1}^{3} \ldots p_{5}^{3} \alpha, P\left|\lambda_{2}\right|^{-1 / 3}\left(p_{1} \ldots p_{5}\right)^{-1}\right)+O\left(1+P^{3 / 2}|\alpha|^{1 / 2}\right)\right) \\
& =\left|\lambda_{2}\right|^{-1 / 3} \Gamma v(-\alpha, P)+O\left(P^{8 / 9}\right) .
\end{aligned}
$$

Here

$$
\Gamma=\prod_{1 \leqslant i \leqslant S} \sum_{P^{\theta i}<p_{i} \leqslant 2 P^{\theta i}} \frac{1}{p_{i}} \asymp(\log P)^{-5} .
$$

For

$$
P^{-5 / 2}<|\alpha| \leqslant P^{-2-\varepsilon}
$$

we claim that

$$
\left|g_{2}(\alpha)\right|<P^{8 / 9} .
$$

For suppose the contrary. By Lemma 5 there are coprime integers $a, q$ with

$$
\begin{aligned}
q & \ll P^{1 / 3}, \\
\left|\lambda_{2} \alpha-a / q\right| & \ll q^{-1 / 3} P^{-26 / 9} \ll q^{-1} P^{-2} .
\end{aligned}
$$

We cannot have $q=1$, since then $a=0$ and

$$
\alpha \ll P^{-26 / 9},
$$

which contradicts (4.5). Consequently we may deduce from (4.7) that

$$
\left|\lambda_{2} \alpha\right|>1 / 2 q \gg P^{-1 / 3} \text {. }
$$

Again, this contradicts (4.5) and we have established (4.6).

Combining (4.2), (4.3)

$$
f_{1}(\alpha) g_{2}(\alpha)=\left|\lambda_{1} \lambda_{2}\right|^{-1 / 3} \Gamma|v(\alpha, P)|^{2}+O\left(P^{8 / 9}(1+|v(\alpha, P)|)\right),
$$

for $|\alpha|<P^{-5 / 2}$, while

$$
f_{1}(\alpha) g_{2}(\alpha)=O\left(P^{8 / 9}(1+|v(\alpha, P)|)\right),
$$


when (4.5) holds. Thus, abbreviating the integrand in (4.1),

$$
\begin{aligned}
& \int_{\mathscr{N}} f_{1} g_{2} h_{3} \ldots h_{7} e(\alpha \mu) K d \alpha \\
& =\left|\lambda_{1} \lambda_{2}\right|^{-1 / 3} \Gamma \int_{|\alpha|<P^{-5 / 2}}|v(\alpha, P)|^{2} h_{3} \ldots h_{7} e(\alpha \mu) K d \alpha \\
& \quad+O\left(P^{8 / 9} \int_{-1}^{1}(1+|v(\alpha, P)|)\left|h_{3} \ldots h_{7}\right| d \alpha\right) .
\end{aligned}
$$

Integration by parts yields

$$
v(\alpha, P) \ll P\left(1+P^{3}|\alpha|\right)^{-1} .
$$

With the trivial bound $h_{j}=O(P)$ we get

$$
\int_{-1}^{1}|v(\alpha, P)|\left|h_{3} \ldots h_{7}\right| d \alpha \ll P^{3} \log P .
$$

From the bound $h_{3}=O(P)$ and

$$
\int_{0}^{1}\left|h_{j}\right|^{4} d \alpha \ll P^{2+\varepsilon}
$$

we get

$$
\int_{-1}^{1}\left|h_{3} \ldots h_{7}\right| d \alpha \ll P^{3+\varepsilon} .
$$

Combining (4.9) and (4.10), the $O$ term in (4.8) is

$$
\ll P^{4-1 / 10} \text {. }
$$

By following through the argument on p. 55 of [3], we find that

$$
\int_{-P^{-5 / 2}}^{P-5 / 2}|v(\alpha, P)|^{2} h_{3} \ldots h_{7} K e(\alpha \mu) d \alpha \gg P^{4-\varepsilon} .
$$

We may now obtain

$$
\int_{\mathscr{N}} f_{1} g_{2} h_{3} \ldots h_{7} e(\alpha \mu) K d \alpha \gg P^{4-\varepsilon}
$$

on assembling (4.8), (4.11) and (4.12). 
§5. The minor arc. Since $K(\alpha) \ll 1$, our Theorem will follow from (4.13) once we show that

$$
\int_{m}\left|f_{1} g_{2} h_{3} \ldots h_{7}\right| d \alpha \ll P^{4-2 \varepsilon}
$$

Let

$$
E=\left\{\alpha \in m:\left|f_{1}(\alpha)\right|<P^{3 / 4+\varepsilon}\right\} .
$$

By Hölder's inequality,

$$
\int_{E}\left|f_{1} g_{2} h_{3} \ldots h_{7}\right| d \alpha \leqslant P^{3 / 4+\varepsilon}\left(\int_{m}\left|g_{2}\right|^{6} d \alpha\right)^{1 / 6} \prod_{j=3}^{7}\left(\int_{m}\left|h_{j}\right|^{6} d \alpha\right)^{1 / 6} .
$$

It is clear that

$$
\int_{m}\left|g_{2}(\alpha)\right|^{6} d \alpha \ll P^{\sigma} \int_{0}^{1}\left|g_{2}(\alpha)\right|^{6} d \alpha
$$

and similarly for $h_{j}$. It follows from Proposition 1 and Lemma 3 that

$$
\begin{aligned}
\int_{E}\left|f_{1} g_{2} h_{3} \ldots h_{7}\right| d \alpha & \ll P^{3 / 4+\varepsilon+\sigma}\left(P^{3+0.25129}\right)^{1 / 6}\left(P^{3+0 \cdot 24957}\right)^{5 / 6} \\
& \ll P^{4-2 \varepsilon}
\end{aligned}
$$

Next, we treat the set

$$
F=\left\{\alpha \in m: P^{3 / 4+\varepsilon} \leqslant\left|f_{1}(\alpha)\right| \leqslant P^{4 / 5}\right\} .
$$

By Hölder's inequality

$$
\begin{aligned}
& \int_{F}\left|f_{1} g_{2} h_{3} \ldots h_{7}\right| d \alpha \ll\left(\sup _{\alpha \in F}\left|f_{1}(\alpha)\right|^{1 / 2}\right)\left(\int_{F}\left|f_{1}\right|^{4} d \alpha\right)^{1 / 8} \\
& \times\left(\int_{m}\left|g_{2}\right|^{8} d \alpha\right)^{1 / 8} \prod_{j=3}^{7}\left(\int_{m}\left|h_{j}\right|^{20 / 3} d \alpha\right)^{3 / 20} \\
& \ll P^{\tau}
\end{aligned}
$$

where

$$
\tau=\frac{2}{5}+\frac{1}{8}+\frac{5}{8}+5 \times \frac{3}{20} \times 3.79417+\sigma<4-2 \varepsilon .
$$

Here we have used the definition of $F$, the analogue of (5.2), Lemma 6, Hua's inequality and Proposition 2. 
It remains to treat those $\alpha$ with $\left|f_{1}(\alpha)\right|>P^{4 / 5}$. We begin with

$$
G=\left\{\alpha \in m:\left|f_{1}(\alpha)\right|>P^{4 / 5},\left|g_{2}(\alpha)\right| \leqslant P^{8 / 9}\right\} .
$$

By Hölder's inequality,

$$
\begin{aligned}
\int_{G}\left|f_{1} g_{2} h_{3} \ldots h_{7}\right| d \alpha & \ll\left(\sup _{\alpha \in G}\left|g_{2}(\alpha)\right|^{2 / 3}\right)\left(\int_{G}\left|f_{1}\right|^{3} d \alpha\right)^{1 / 3} \\
& \times\left(\int_{m}\left|g_{2}\right|^{8} d \alpha\right)^{1 / 24} \prod_{j=3}^{7}\left(\int_{m}\left|h_{j}\right|^{8} d \alpha\right)^{1 / 8} \\
\ll &
\end{aligned}
$$

where

$$
v=\frac{16}{27}+\frac{1}{15}+\left(\frac{1}{24}+\frac{5}{8}\right) \times 5+\sigma+\varepsilon<4-2 \varepsilon .
$$

Here we have used the definition of $G$, the analogue of (5.2), Lemma 7 and Hua's inequality.

The set which remains is

$$
H=\left\{\alpha \in m:\left|f_{1}(\alpha)\right|>P^{4 / 5},\left|g_{2}(\alpha)\right|>P^{8 / 9}\right\}
$$

and it is at this stage that we must restrict $P$ to the values

$$
P=q^{2 / 3}
$$

( $q$ a denominator of a convergent to the irrational number $\lambda_{1} / \lambda_{2}$ ). We split $H$ into $O\left((\log P)^{2}\right)$ sets

$$
H(A, B)=\left\{\alpha: A<\left|f_{1}(\alpha)\right| \leqslant 2 A, B<\left|g_{2}(\alpha)\right| \leqslant 2 B\right\}
$$

with $P^{4 / 5} \leqslant A \leqslant P, P^{8 / 9} \leqslant B \leqslant P$. Because of the Diophantine approximation provided by Lemmata 4 and 5, we may follow the argument in Baker [1], pp. 89-90 to bound the Lebesgue measure of $H(A, B)$ by

$$
\ll P^{9 / 4+7 \sigma / 6+\varepsilon}(A B)^{-7 / 2}
$$

Consequently, with trivial bounds on $h_{j}$,

$$
\int_{H}\left|f_{1} g_{2} h_{3} \ldots h_{7}\right| d \alpha \ll P^{5+9 / 4+2 \sigma}(A B)^{-5 / 2} \ll P^{7 / 2} .
$$

We have established (5.1), and the Theorem follows.

Acknowledgement. R. C. Baker thanks the US NSF for a grant. T. D. Wooley thanks the U.S. NSF for a grant and is also grateful for an Alfred P. Sloan Research Fellowship and Fellowship from the David and Lucile Packard foundation. 


\section{References}

1. R. C. Baker. Cubic Diophantine inequalities. Mathematika, 29 (1982), 83-92.

2. J. Brüdern. Additive Diophantine inequalities with mixed powers I. Mathematika, 34 (1987), $124-130$.

3. J. Brüdern. Cubic Diophantine inequalities. Mathematika, 35 (1988), 51-58.

4. J. Brüdern. A note on cubic exponential sums. Sém. Théorie des Nombres, Paris, 1990-1991, 23-24, (S. David, ed.), Progr. Math.

5. H. Davenport. On indefinite quadratic forms in many variables. Mathematika, 3 (1956), 81 101.

6. H. Davenport and K. F. Roth. The solubility of certain Diophantine inequalities. Mathematika, 2 (1955), 81-96.

7. Ju. V. Linnik. On the representation of large numbers as sums of seven cubes. Mat. Sbornik, 12 (1943), 218-224.

8. R. C. Vaughan. The Hardy-Littlêwood method (Cambridge University Press, 1981).

9. R. C. Vaughan. Some remarks on Weyl sums. Colloq. Math. Soc. Janos Bolyai, 34 (Elsevier, North-Holland, Amsterdam 1984), 1585-1602.

10. R. C. Vaughan. On Waring's problem for cubes. J. Reine Angew. Math., 365 (1986), 122-170.

11. R. C. Vaughan. A new iterative method in Waring's problem, Acta Math., 162 (1989), 1-71.

12. R. C. Vaughan. On Waring's problem for cubes II. J. London Math. Soc. (2), 39 (1989), 205218.

13. T. D. Wooley. Breaking classical convexity in Waring's problem: sums of cubes and quasidiagonal behaviour. Inventiones Math., to appear.

Professor R. C. Baker,

Department of Mathematics,

Brigham Young University,

Provo, UT 84602,

U.S.A.

Dr. J. Brüdern,

Mathematisches Institut A,

Postfach 80-11-40,

Universität Stuttgart,

D-7051, Stuttgart,

Germany.

Professor T. D. Wooley,

Department of Mathematics,

University of Michigan,

Ann Arbor, MI 48109-1003,

U.S.A.

\section{D75: NUMBER THEORY: \\ Diophantine equations; \\ Diophantine inequalities.}

Received on the 12th of October, 1994. 